\title{
Teaching Research Ethics on Clinical Trials to Multidisciplinary and International Trainees in Global Infectious Disease Research
}

\author{
Julius Oyugi*, Cisily Meeme, Nadia Chanzu, Julie Ambia, Jesse Gitaka, Frank Ndakala, Melissa Herman, Lyle Mckinnon, Joseph Kaufert \\ and Keith Fowke \\ University of Nairobi, Nairobi, Kenya
}

The term global health is used to stress health issues that transcend national borders, class, race, ethnicity, income, and culture. Such global health issues result in the need for development of drugs and vaccines, which in turn demand ethical recruitment and informed participation of sufficient numbers of participants for clinical trials [1]. The number of clinical trials continues to rise as evidenced by their registration at repositories $[2,3]$.

A critical component of clinical trials is the need to understand the historical and philosophical basis for applying bioethical principles; understanding research ethics codes and guidelines and interpreting and applying regulations and policy frameworks in the design and actual conduct of research [4]. Frameworks for protection of human subjects must both assure ethical recruitment and treatment of participants while ensuring research questions are addressed effectively $[5,6]$. The primary investigator thus needs training to gain capacity to tackle the myriad of ethical dilemmas that emerge during trials. Majority of the training programs currently available are based within existing research projects and do not expose the trainees to the multicultural and multidisciplinary reality on the ground. This type of training limits the trainees' exposure to a host of potential issues that may arise in different real-world study scenarios. Knowledge exchange that traverses different study settings is an important aspect of training in clinical trials needs to be considered when training futures clinical trials investigators.

In line with this, IID \& GHTP, a multidisciplinary research-training program funded by the CIHR organized a two-week course that was designed to provide an overview of the design, implementation and ethical issues involved in conducting clinical trials. The focus of the training was to discuss the ethical challenges of conducting clinical trials in a developing country. Core modules in the first two days of the course focused on providing trainees with both the philosophical basis and current policy frameworks for applying internationally were addressed. The introduction emphasized that principals such as autonomy achieved through research consent agreements were the foundation for ethical research relationships. As such they modeled ethical conduct within a legal and philosophical context and enabled research to be formally evaluated and monitored by IRBs. However modules presented later in week 1, case examples of Kenyan trials (such as the trial of male circumcision) and also the field experience in week 2 introduced problematic areas or ethical dilemmas emerging from actual research practice. For example, problems such as the increasing complexity of consent agreements required in multicentre and multinational trials and the use of oral consent were introduced as "dilemmas" which required both additional cultural and ethical resolution before a formal policy statement and framework for protection of research participants within the trial could be developed.

The modules utilized pedagogical approaches (e.g. case studies, small group problem solving exercises and policy debates) developed during three previous Indiana University/ Moi University (TaSkR) workshops on 'Teaching Skills in International Research Ethics' [7].
These introductory sessions on theory and current research ethics practice were reinforced by including infectious disease research in international and developing countries. Examples of applications of research ethics in collaborative international research in Kenya were used. These sessions were linked with the portions of the course dealing with trial design, analysis and implementation.

\section{Objectives}

The objective of this report was to inform the international community on the successes and challenges experienced in conducting a training program on research ethics in clinical trials for international trainees with multidisciplinary backgrounds. We report a number of key issues that arose due to the uniqueness of this training program, and may serve as strong reference to guide the planning and implementation of similar training programs in the future.

\section{Approaches}

The IID \& GHTP 2012 clinical trials and research ethics course was held at the Kenya AIDS Vaccine Initiative (KAVI), University of Nairobi, Kenya. The training brought together trainees from different continents with different cultural backgrounds. All trainees had a general background in infectious diseases but with specific backgrounds in basic science, social science, epidemiology and clinical science and varied levels of knowledge on ethical conduct of research. More than half of the trainees had participated in brief modules describing contemporary issues in research ethics in the introductory course. The participants included doctoral students, postdoctoral fellows and clinical fellows from Canada, Colombia, Kenya and India. Also included were clinical trials physicians, nurses and project managers from different study projects within Kenya. The diversity of clinical trials in Kenya provided a rich environment for the trainees to be exposed to different clinical trials scenarios in a developing country, with a heavy infectious disease burden. The training sites were chosen on the basis of their uniqueness in terms of the type of clinical trials being conducted as well as their unique cultural setting. The clinical trials sites included: (i) A clinical trial on prevention of bacterial vaginosis in Nairobi, (ii) An HIV vaccine clinical trial in Nairobi, (iii) HIV pre-exposure prophylaxis trials in the towns of Thika and Bondo, (iv) A clinical trial on malaria prevention in pregnancy in Siaya

*Corresponding author: Julius Oyugi, University of Nairobi, Nairobi, Kenya, Tel Aviv, 64239, Israel, Tel: 254-20-2726300 extn 43163; E-mail: researchdept10@gmail.com

Received November 03, 2013; Accepted December 28, 2013; Published January 14,2014

Citation: Oyugi J, Meeme C, Chanzu N, Ambia J, Gitaka J, et al. (2014) Teaching Research Ethics on Clinical Trials to Multidisciplinary and International Trainees in Global Infectious Disease Research. J Clin Res Bioeth 5: 166. doi:10.4172/21559627.1000166

Copyright: ( 2014 Oyugi J, et al. This is an open-access article distributed under the terms of the Creative Commons Attribution License, which permits unrestricted use, distribution, and reproduction in any medium, provided the original author and source are credited. 
town and (v) A clinical trial on integration of antenatal care and HIV prevention in Migori town.

Modes of delivery for the training included a blend of lectures and case scenarios, group/panel discussions, field visits/assignments and designing hypothetical clinical trials that could be conducted at each site. The lectures were delivered by both local and international experts in the fields of research ethics and clinical trials, emphasizing internationally recognized principles, compliance with international, national and local research codes and policy regulations. Both structured presentations and problem-solving experiences emphasized how frameworks for protection of human subjects could be applied to clinical trials on a local, national or global scale.

The training was structured into two parts. Part I consisted mainly of providing students with an introduction to research ethics as applied in clinical trials. It covered key concepts and principles, procedures for ethical review boards and challenges they face, considerations for conducting trials as well as obligations to the community following trial completion. This part of the course was designed to provide the trainees with enough basic knowledge on ethical considerations and clinical trials to allow them to effectively complete the second part of the course.

Part II involved trainee-led practica at the aforementioned clinical trials sites. Each site was assigned a mentor and the trainees spent 4 days on site gathering information and making observations on the ongoing clinical trials. The trainees were also assigned a hypothetical clinical trials task where they were then expected to design a clinical trial, based on following topics: (i) A genetically modified lactobacillus for bacterial vaginosis treatment, (ii) Evaluation of gene therapy as an HIV treatment, (iii) HIV prevention through behavioral intervention, (iv) A phase $3 \mathrm{HIV}$ vaccine trial and (v) Increasing adherence to HIV prevention and long acting pre-exposure vaginal gel.

For the final 2 days of the course, trainees assembled in Nairobi to make presentations on their observations and practica assignments before fellow trainees and course trainers. Originality of proposed hypothetical trials, design, ethical considerations, and relevance to community needs were assessed.

\section{Course structure and content}

Four key themes were covered in this training programme: (i) Experience of integrated multidisciplinary training, (ii) Community based research and the role of community based research workers, (iii) Trial considerations, conduct and monitoring and iv) Practical experience through field visits and clinical trial design.

Experience of integrated multidisciplinary training: A team of research experts from Canada, USA and Kenya were brought together to share their knowledge, expertise and experience in working in the area of infectious diseases in reference to clinical trials. Their varied fields of specialization and experience that included medical microbiology, bioethics, medical anthropology, medicine, statistics, and epidemiology offered trainees an opportunity to see the intersection of basic science, social science and epidemiology and their relevance for successful clinical trials.

Trainees were able to gain an understanding of the differences between individual rights and collective rights in research ethics focusing on the control of infectious diseases. They were also introduced to the arguments of ethical reasoning and how to draw a distinction between research ethics, public health ethics and clinical ethics as they impact population research, clinical trials and health services or public health research.

Through group deliberations of case studies trainees were able to identify areas of variation in the process of obtaining informed consent in North America, Africa and other global research environments. They were further able to interpret the challenges of maintaining informed consent from individuals and communities and how to engage with problems of obtaining meaningful informed consent in cross-cultural and cross-national studies $[8,9]$.

Trainees were provided with an understanding of the regulatory oversight in cross-national and multisite research. This included an overview of the challenges that researchers face in harmonization of different codes, guidelines, and declaration regulations [10]. The trainers drew examples on the current research ethics policy and review systems in Kenya and other alternative models of research ethics review bodies using case material from indigenous ethics review boards and private ethics review boards. The importance of research ethics from the perspective of the participant, the researcher, sponsoring institution, ethics review boards and community or national governments was underscored.

Community based research and the role of community based research workers: Aspects of community engagement in clinical research were extensively covered, with trainees learned the importance of balancing community and researcher expectations. Emphasis lay on the ethical issues that are supposed to govern the processes and the role of ethical oversight of community research workers and models for engaging community and individual consent. The training drew examples from the KAVI HIV vaccine trials, the case of community participation and the role of community level research in Randomized Controlled Trials (RCTs). An example was also presented in a case describing the trials of male circumcision on HIV transmission in Kenya.

Another case example in the course described a malaria intervention trial with reference being on the ability of women to provide informed consent and some of the unique challenges women face in this context. Drawing from global perspectives, it was noted that research ethics is both contextual and dynamic. For example, women in Kenya, like women and children in many societies, are considered to be vulnerable, thus diminishing their autonomy to make informed decisions about their participation in trials. However this vulnerability may not be universal among the heterogeneous subpopulation of Kenyan women. Therefore the capacity of a female research participant to make informed, independent decisions might be influenced by a number of contextual factors that include her culture, class, education, and religion and family relations.

Trainees were able to gain an understanding of mechanisms for engaging with and developing frameworks for ethical protection of the communities. In addition to developing consent and ethical protection documentation for individuals, frameworks for protecting communities involved and other collectivities (e.g. sex workers) in research from exploitation and unethical recruitment are essential in order to develop and sustain long-term research relationships with individuals and communities.

Trial considerations, conduct and monitoring: Trainees were introduced to ethical rules and considerations before, during and after clinical trails with examples of available literature. An overview of the phases of clinical trials and their relevance was given. This included 
the different aspects of trial designs and their applicability to different trials. The trainees were further introduced to statistical considerations for clinical trials. Factors to consider for sample sizes were discussed and this included power calculations and the selection of endpoints. Central to good clinical practices is conduct of ethics, so drawing from Kenyan experiences; trainees were taken through the processes of trial conduct and monitoring. Drawing from the KAVI experiences, the trainees learnt about the processes of acquiring ethical approval for trials and research conduct, including actions taken in the event of occurrence of unexpected outcomes, such as serious adverse events and proper documentation and reporting of these events.

Aspects relating to trial conclusion, the processes of clinical data management and analysis, the roles of the data monitor, sharing results and publications and post-trial obligations were discussed. Finally, the trainees were made aware of the various milestones that have been achieved in addressing community benefits in clinical trials including legal redress.

Practical experience through field visits and clinical trial design: Following the lectures, the trainees were given an opportunity to visit clinical trial sites in Kenya. The objectives of these visits were to enable them to get first hand contact with existing clinical trials, learn how different trials are conducted and based on their learning experience in the field, understand the basics of how to design trials. Trainees were given the opportunity through this section of the course to spend time reviewing the current or recently completed clinical trial at their assigned site, with access to staff, coordinators, and sometimes, participants, to gain a broad understanding of the framework and inner workings of the trial. After this initial analysis, trainees were given the opportunity to design their own clinical trial based in the site in question, giving them the chance to put to work the knowledge and skills they had gained during the classroom learning portion of this course. With that knowledge, trials were designed keeping in mind ethical considerations, cultural and community interactions, informed consent, and the proper way to structure and format a real-world clinical trial within a low-income setting. Overall, it was felt that this format of combining classroom learning with hands-on experience developing a new clinical trial was a unique method which afforded trainees the opportunity to gain the base knowledge required while also getting a chance to acquire real-world clinical trials experience.

\section{Relevance and suitability of the 'Clinical Trials and Research Ethics' training program in a limited-resource setting}

The relevance and suitability of this course for its target audience and for a limited-resource setting are evaluated based on content of the course and structure, training tools and methods used as well as effectiveness of the faculty.

Course content and structure: Topics from two arms were covered within the course: clinical trials and ethics. The program was structured so that foundation topics such as history, key concepts, principles and design were offered first before moving on to more complex issues related to the application and implementation of these topics in clinical trials, while ensuring congruence in research objectives and community interests. Case studies were presented and classroom group discussions were set up to better aid in understanding these topics. Trainers gave candid accounts and examples of their experiences in past and present clinical trials as a method of supplementing the information presented.

Trainers and instructors: Trainers and instructors were drawn from diverse backgrounds and nationalities and are respected authorities in their respective fields. Instructors came from The Kenya AIDS Vaccine Initiative (KAVI), University of Nairobi, Kenya, Moi University, Kenya, The Kenya National Ethics Board, and Center for Bioethics, of Indiana University, USA, University of Toronto, Canada, Johns Hopkins University, USA, and Illinois University, USA. Background experience of trainers and instructors included bioethics and moral philosophy as well as applied experience in running clinical trials, monitoring and evaluating clinical trials, developing and participating in reviews by ethical review boards and consultation with community engagement workgroups.

Clinical trial field assignments: Teams were made up of trainees from Canada, Colombia, Kenya and India, with basic science, epidemiology and social science backgrounds, along with one experienced clinical 'trialist' from a participating research site. This diversity translated into a diverse array of perspectives on ethics in clinical research and provided for very constructive and informative discussions. Trainees were able to appreciate the dynamics of engaging the community in study design and the importance of designing studies with both practical and comprehensive aims.

The diversity of trainers was effective in presenting a clear picture of the challenges and merits involved in conducting clinical trials in resource-rich versus limited-resource settings. It also highlighted the intricacies of navigating collaborative research between groups from the two resource spectra and the delicate balance of ensuring research objectives are met while respecting community interests. While local trainers were effective in dissecting ethical dilemmas with a strong grasp of the local community's cultural practices and knowledge, visiting trainers enriched the course by providing independent viewpoints. We feel it is prudent this fact be taken into account when designing future courses in limited-resource and multicultural settings.

When an invitation was extended to participating research sites to send one member of staff to the training, all sites were willing to send more people but were locked out due to unavailability of positions. This served as an indicator of the need and relevance of this course in Kenya, and by extension, potentially within other limited-resource countries. Having identified this gap, KAVI has taken the initiative to address it. KAVI, within the auspices of The Regional AIDS Training Network (RATN), has adopted the 'Clinical Trials and Ethics course' concept, format and curriculum to develop an annual training programmed for the Eastern and Southern Africa region.

Based on trainee evaluations during and after the course, trainees were satisfied with the structure of the course, and found the clinical trial site visits and opportunities to develop their own clinical trial to be very beneficial. Trainees felt that the visits to field sites impacted their knowledge base with the evaluations on the field visits scoring highly (0.84-1.00)

“...As a PhD student working on a basic science project, my experience in other aspects of health research, such as clinical trials, social science, and even ethics, is very minimal. During the Clinical Trials and Ethics course in Nairobi, Kenya in February 2012, I was afforded the opportunity to greatly broaden my knowledge of clinical trials by being directly immersed in the inner workings of one such trial. The Clinical Trials course was a combination of classroom learning and on-site, realworld experience, which provided us the chance to come away with a new and valuable skill set."

\section{-Melissa, Canadian trainee.}

Trainees reported that they found the course relevant and even found it applicable in their respective disciplines. 
"...Back in India, I am associated with an RCT that tests the effectiveness of onsite mentoring intervention at primary health care centers in improving the quality of maternal and newborn services. Being the principal investigator, I was involved in developing of protocols and tools, consent forms and ethical board submission. The knowledge about ethical issues that was learnt during the course was very useful during this time."

\section{-Krishnamurthy, Trainee from India.}

\section{Successes and challenges of the 'Clinical Trials and Research Ethics' training program}

Though the course was a great success, there were a number of challenges experienced, owing to the diversity of trainers and trainees and the novelty of the training program.

Knowledge of issues with ethical implications in clinical trials: The ethicists and educators mainly highlighted the use of a structured approach to ethical decision-making to assist trainees in making sense of complex ethical issues. However, one of the key challenges faced was that the students were from multidisciplinary backgrounds, such that in some cases, in relation to clinical trials research, the students lacked the familiarity with the science behind an issue with ethical implications. This hindered their analysis of a number of issues presented and reflected in their drawing of conclusions. However, the presence of a number of trainees with strong backgrounds in basic science, those trainees from other backgrounds were able to lean on the understanding of the basic scientists to clarify some of the science behind these issues. In future, it may be beneficial to provide a brief, layman-style overview of the key basic science concepts that underscore some of the ethical considerations in clinical trials for a course of this nature. The facilitators might also need to administer a pretest survey prior to the course, to determine attendees' level of knowledge thus customize training content.

African versus western cultures: bias in the critical analysis: The teaching of research ethics is often reinforced with the use of illustrative case studies. This approach is ideal, as it allows the use of present examples demonstrating a range of significant ethical dilemmas related to clinical trials and the complexities associated with each of the issues. During this course, the cases presented were based on actual clinical trials conducted across continents, and we recognized a bias in the critical analysis of the ethical complexities as analyzed by the students, when comparing studies on infectious diseases in an African versus the non-African environment. While this was a challenge, it was also seen as an opportunity for trainees from such vastly different backgrounds to share their opinions and viewpoints, and learn from one another in culturally relevant ways. A course of this kind, which brings together students from such diverse backgrounds, allows for a sharing of knowledge, experiences, and culturally sensitive perspectives, which, while opinions may have differed, served to enrich the overall experience.

Complexity of real-life situations: This course was designed for doctoral and postdoctoral research trainees specializing in basic, social and epidemiological sciences. In this regard, it has been shown that it is difficult to promote ethical practice solely through education, as there is often limited practical value of the assignments that comprise formal assessment. While the ethics principles and concepts taught during this course assisted in the students' ability to resolve ethical dilemmas, in real-life situations, these principles are often in conflict. More often than not, when students graduate to become professionals in their different fields of expertise, they may find it difficult to determine which principles to prioritize in particular cases. However, by providing trainees with as many real-world examples as possible, and by giving them the basics of how to build an ethical framework for clinical trials, it gives them a basis from which to work from. These principles, though details may differ in real-world scenarios, are still applicable and a good starting point from which the students can work in their future careers. In order to keep abreast trainees should be encouraged to enroll for online research ethics courses designed by reputable organizations.

Protection of vulnerable population-ethical consideration: In the introductory modules the issues of ethical protection for vulnerable populations in global health research was raised in terms of both protection of at risk groups. The vulnerable groups included, (i) adolescent participants under the legal age of consent, (ii) persons with sensory and intellectual disabilities and (iii) other groups defined in terms of gender or genetically defined risk factors. Presenters from bioethics emphasized the need to develop special protections for vulnerable individuals and groups and also the need to include vulnerable populations in human subject populations to insure both just representation and potential benefits of research to members of these populations. For example in field practicum experience trainees worked with programs that developed community advisory committees and alternative assent agreements to enable adolescents to participate in trials and descriptive research.

Ethicists, not scientists, as research ethics trainers: The need for explicit training in research ethics for scientists is widely recognized, and although many science experts are keen on including research ethics in their curricula, they may lack the expertise to conduct training on research ethics. Therefore, this session highlighted the urgent need for ethicists and educators, as they have an added advantage and experience in designing curricular materials for conducting workshops and teaching research ethics. The challenge in having enough expert facilitators was however overcome by inviting experts from across the globe to facilitate the sessions. Following the success of the training program, we are optimistic that faculty who participated may be able to design workshops integrating fundamental issues in research ethics. The faculty had an opportunity to gain skills in teaching research ethics, and the institutions as a whole will build a greater commitment to and expertise in incorporating research ethics in syllabi.

Interactive sessions: This was the first research ethics session conducted among the trainees. The curriculum lectures and facilitator notes outweighed the case study interactive sessions. This was quite a challenge as the students tended to be less interactive and towards the end of the course, mentally fatigued due to the intensity of the course notes. It may prove more effective to teach research ethics by encouraging students to participate in active learning. This is advantageous as it provides opportunities for students to listen to alternative views, discuss their own views, debate amongst themselves, and apply critical reasoning to complex situations. Occasionally the students may present their own work, and recount their own experiences in obtaining research ethics approval from their local research ethics boards even as analysis of case studies is be used to enhancing understanding. These tactics help to ensure that trainees are always engaged in the learning process and we feel helps to solidify the learning.

Assessment strategies: Evaluating a trainee's understanding of research ethics can be quite a challenge. This is because there may be a key focus on the application of research ethics as opposed to 
Citation: Oyugi J, Meeme C, Chanzu N, Ambia J, Gitaka J, et al. (2014) Teaching Research Ethics on Clinical Trials to Multidisciplinary and International Trainees in Global Infectious Disease Research. J Clin Res Bioeth 5: 166. doi:10.4172/2155-9627.1000166

Page 5 of 5

the comprehension of the paradigms of research ethics. During this session, assessment was based on the hypothetical trials designed at the end of the course as a method of evaluating the trainees' understanding of the structure, formation and interaction of ethics and clinical trials. However, there are a number of additional approaches that should be considered, at different stages of the course. These include critical analysis of published papers, short answer questions at the end of lectures and/or extended questions evaluating the trainees understanding on the mechanism and process of ethical reviews. Roleplays (as members of ethics research boards), active discussions and peer assessments may also be considered. These sessions allow the students to undertake active learning.

\section{Conclusion and Recommendations}

The training course succeeded in exposing trainees to the many challenges of conducting ethically sound research that is mutually beneficial to both the researcher and the community involved. The format and content of the training course and challenges faced can be attributed to the novelty of the program. This course provided an overview of clinical trials, with a focus on the ethical issues surrounding the proper execution of clinical trials in the developing world. The training program also provided a platform specifically designed to promote debate and awareness on emerging ethical issues in international collaborative health research between developing and developed countries.

We recommend that other local institutions adopt such training for different groups such as clinicians, with refresher courses as needed, to keep them up to date as ethics and clinical trials grow and develop. There is also a need to include tools to assess performance of the training program from the viewpoint of both the trainees and the trainers, from the start to the end of the training period.

\section{References}

1. Edwards R, Piachaud J, Rowson M, Miranda J (2004) Understanding global health issues: are international medical electives the answer? Med Educ 38 688-690.

2. The Pan African Clinical Trials Registry (PACTR-2013).

3. United States National Library of Medicine(USNLM-2013) Clinical trials registry

4. Kessel F and Rosenfield PL (2008) Toward transdisciplinary research Historical and contemporary perspectives. American Journal of Preventive Medicine 35: 225-234.

5. Emmanuel EJ, Wendler D, Killen J,Grady C (2004) What makes clinical research in developingcountries ethical? The benchmarks of ethical research. The Journal of Infectious Diseases 189: 930-937.

6. Emmanuel EJ, Wendler D, and Grady C (2000) What makes clinical research ethical? JAMA: the journal of the American Medical Association 283: 2701 2711.

7. Meslin E (2011) Teaching Skills in International Research Ethics Workshop TaSkR III: Program and Objectives, Centre for Bioethics, Indiana University, Indianapolis, Indiana, 1-201.

8. Brewster D (2011) Science and ethics of human immunodeficiency virus/ acquired immunodeficiency syndrome controversies in Africa. Journal of Pediatrics and Child Health 47: 646-655.

9. Estape E, Laurido LE, Shaheen M, Quarshie A, Frontera Wet al.(2011) A multiinstitutional, multidisciplinary model for developing and teaching translational research in health disparities. Clinical and Translational Research 4: 434-438.

10. King G, Servais M, Forchuk C, Chalmers H, Currie M, et al. (2010) Features and impacts of five multidisciplinary community-university research partnerships. Health and Social Care in the Community 18: 59-69. 